\title{
An in-depth look at how physical properties of cleaning materials affect the removal of soot from rough papers
}

Teresa Duncan ${ }^{1}$, Edward Vicenzi ${ }^{2}$ and Shannon Brogdon-Grantham ${ }^{3}$

${ }^{1}$ Museum Conservation Institute, Smithsonian Institution, Suitland, Maryland, United States, ${ }^{2}$ Smithsonian Institution, Museum Conservation Institute, Suitland, Maryland, United States, ${ }^{3}$ Museum Conservation Institute, Smithsonian Institution, United States

Soot is notoriously challenging to remove from surfaces due to its small (nanoscale) size and low polarity. ${ }^{1}$ Although general guidelines for the removal of soot have been provided to art conservators, there has been no widespread comparative study to supply paper conservators with the information necessary to choose treatment methods best suited to archival collections. In an effort to gather information to aid conservators in the selection of cleaning materials for soot removal, we have performed a survey comparing ten different methods for the cleaning of lamp black (a form of soot prepared with modern oil furnaces typically used as a black pigment) from papers with differing degrees of roughness. The cleaning methods explored include traditional (a dry swab; a cosmetic sponge; a soot sponge; a Wishab dry cleaning sponge; eraser crumbs; Absorene Paper, Book, and Paper Wallpaper cleaner; and Magic Rub \#1954, art gum, and kneaded erasers) and one recently-proposed technology (a solvent-free Sylgard 184 elastomer that can remove particulates with a gentle 'press and peel' action $\left.{ }^{2,3}\right)$. The visual changes to the paper substrates with cleaning are documented with two macroscopic techniques: photodocumentation of the paper substrates after cleaning (Figure 1) qualitatively illustrated the overall cleaning results and colorimetry measurements quantitatively served to determine cleaning efficacy. To gain a more in-depth understanding into each method's success for the removal of soot, microscopic analyses of the substrates before and after cleaning provide further information regarding the outcome of various cleaning treatments.

The microscopic examination of select substrates before and after select cleaning methods has explored three questions: 1) how do the physical properties of cleaning materials influence the location of residual soot on the substrate? 2) how does the roughness of the paper substrate dictate the efficacy of each method? and 3) what physical changes occur to the substrate with cleaning? A portable optical microscope has been used to collect images (Figure 2) that aid in the assessment of each cleaning method in relation to: 1) the ability to remove soot from topographically-complex substrates and 2) the propensity to push particles deeper into the substrate. Visual comparisons of the surfaces after cleaning have been supplemented with more in-depth analyses probing the quantity, size, shape, and orientation (relative to orientation of paper fibers) of soot aggregates remaining on the surface after cleaning. Microscopy was also used to investigate any physical changes (such as damaged fibers or residue remaining after treatment) that may be caused by the studied cleaning methods.

Specifically, we have been able to show how soft materials (e.g., soft sponges and putty-like materials) can conform to rough surfaces for more effective cleaning. Firm cleaning materials (e.g., vinyl erasers) can be very effective for cleaning relatively flat areas of paper but are less effective for the removal of particles resting against paper fibers. 
Although tacky materials (e.g., kneaded erasers) are very effective for the removal of lamp black particles from the paper substrate, these materials have shown to pull paper fibers from the surface and their use may be inappropriate on delicate papers. $^{4}$

The macrographs and micrographs collected for this study work in concert to detail which methods are the most successful for removing lamp black from paper and how these methods function to be the most effective. By studying an array of cleaning materials with various physical attributes, we have been able to correlate certain physical properties of the cleaning material swith cleaning efficacy.

\section{Acknowledgements}

T.T.D. would like to acknowledge the Smithsonian Institution for support via the Smithsonian Postgraduate/postdoctoral Fellowships in Conservation of Museum Collections Program.

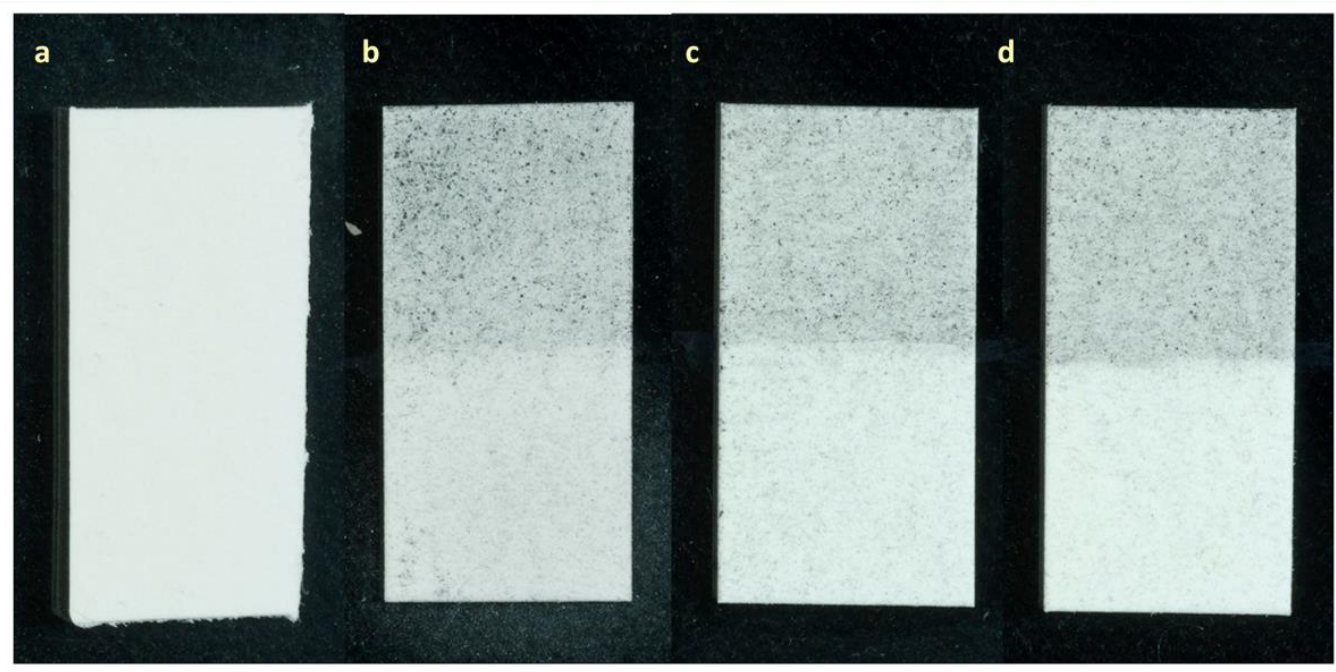

Figure 1. Photographs of rough watercolor paper samples before coating with lamp black (a) and after coating with lamp black and cleaning with a soot sponge (b), a white vinyl eraser (c), and a kneaded eraser (d). In b-d, top half of paper samples left uncleaned to serve as a visual reference. Paper samples are approximately $2.5 \mathrm{~cm} \mathrm{x} 1.3 \mathrm{~cm}$.

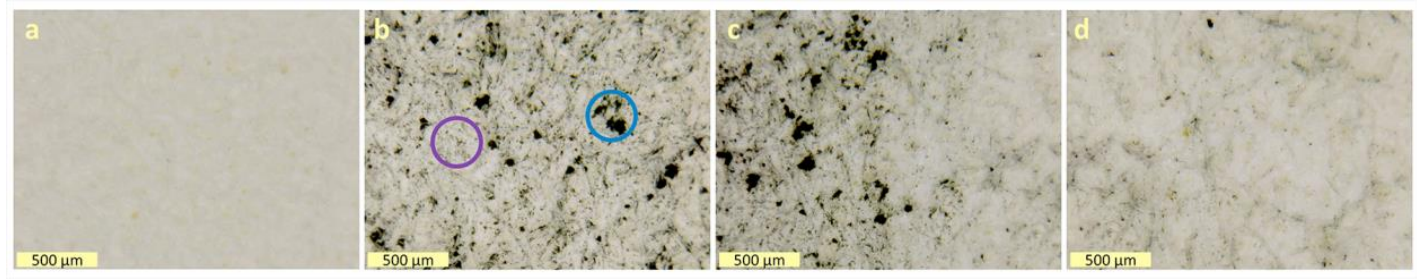

Figure 2. Microscope images collected of rough watercolor paper before (a) and after (b) coating with lamp black. Purple and blue circles show examples of areas with finely dispersed soot and large aggregates, respectively. After cleaning with a white vinyl eraser, images were collected at the interface between 'cleaned' and 'soot-coated' (c) and in the 'cleaned' area $(\mathrm{d})$.

\section{References}


(1) Bolstad-Johnson, D. The Hidden Hazards of Fire Soot. AIC News. 2010, pp 1-5.

(2) Duncan, T. T.; Chan, E. P.; Beers, K. L. Maximizing Contact of Supersoft Bottlebrush Networks with Rough Surfaces to Promote Particulate Removal. ACS Appl. Mater. Interfaces2019, 11 (48), 45310-45318. https://doi.org/10.1021/acsami.9b17602.

(3) Duncan, T. T.; Chan, E. P.; Beers, K. L. Quantifying the Press and Peel Removal of Particulates Using Elastomers and Gels. J. Cult. Herit. In press. https://doi.org/10.1016/j.culher.2020.11.004.

(4) Pearlstein, E. J.; Cabelli, D.; King, A.; Indictor, N. Effects of Eraser Treatment on Paper. J. Am. Inst. Conserv.1982, $22(1), 1-12$. 